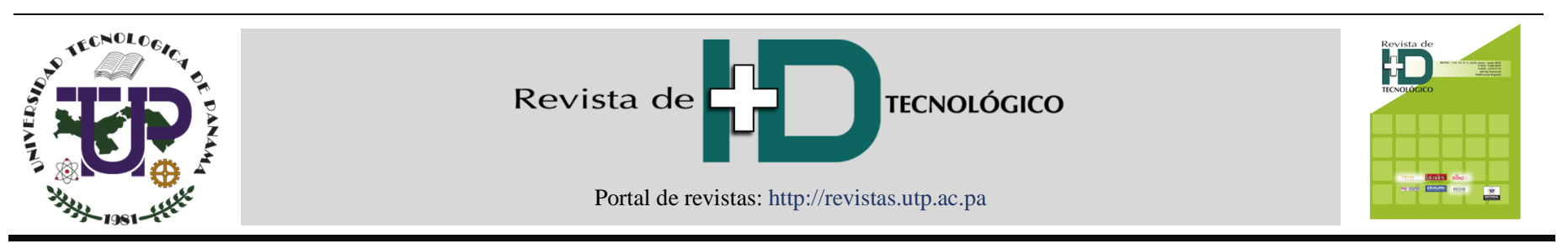

\title{
Evaluación de la experiencia de usuario en el contexto cubano
}

\section{User Experience evaluation in Cuban context}

\author{
Raúl Cuervo Bello ${ }^{1 *}$ \\ ${ }^{1}$ Empresa de soluciones informáticas (Desoft División Santiago), Cuba \\ *Autor de correspondencia: rcuervo@nauta.cu
}

RESUMEN_ La usabilidad puede ser expandida utilizando las posibilidades que ofrece el estudio de la experiencia de usuario. Existen métodos de evaluación dirigidos a la comprobación de la satisfacción del usuario, que utilizan la experiencia, los valores cognitivos y elementos subjetivos. En Cuba se implementan procesos de informatización, los cuales son regulados a través de la gestión de la calidad en software. Esta es comprobada por entidades certificadoras. Estas basan sus fundamentos en políticas, disposiciones legales, normas y modelos establecidos. En este trabajo se revisaron métodos de evaluación de la usabilidad y de la experiencia de usuario, para los productos y servicios informáticos cubanos. Los resultados obtenidos muestran el estado del arte de este tema en el país.

Palabras clave-Calidad en software, experiencia de usuario, métodos de evaluación, usabilidad.

\begin{abstract}
Usability can be expanded using the possibilities offered by the study of User Experience. There are measuring methods aimed to user satisfaction, using experience, cognitive values and subjective elements. In Cuba, informatics processes are implemented. They are regulated through software's quality management, which is verified by certifying entities. Their foundations rely on policies, legal provisions, norms and established models. In this paper, methods of evaluation of Usability and User Experience were reviewed for Cuban IT products and services. The results obtained, show the state of the art of this subject in the country.
\end{abstract}

Keywords-Software quality, user experience, evaluation methods, usability.

\section{Introducción}

La usabilidad aporta conceptos y métodos para desarrollar productos y servicios que alcancen $\mathrm{y}$ mantengan parámetros aceptados como complementos de la calidad [1]. Constituye una guía indispensable para los especialistas que producen y evalúan soluciones informáticas, con el objetivo de comprobar que el producto pueda ser utilizado por los usuarios con efectividad, eficiencia y satisfacción [2].

La experiencia de usuario (UX, del inglés user experience) extiende el alcance de la usabilidad al enfocar sus objetivos en el análisis de factores subjetivos [3], los que se manifiestan en las personas que interactúan con los sistemas.

Los métodos de evaluación centrados en el usuario, permiten medir el grado de satisfacción de los clientes [4], y asegurar la elaboración de características que respondan a los requisitos planteados. Su inclusión desde las primeras fases de desarrollo del producto, contribuye a la reducción de costos [5], y al alcance de metas más realistas en tiempos óptimos.

En Cuba se han implementado políticas que tributan al cumplimiento de planes de desarrollo económico y social, [6, 7], donde la informatización de los procesos posee carácter prioritario [8].

Para la ejecución de estas tareas de manera segura y sostenible, se aplican mecanismos de gestión de la calidad [9], necesarios para garantizar el cumplimiento de estándares internacionales.

El objetivo de este trabajo consiste en analizar cómo se manifiestan las prácticas asociadas a la evaluación de la experiencia de usuario en el país. Se seleccionaron entidades dedicadas a la evaluación y certificación de servicios y productos de software, para analizar los métodos de chequeo empleados. También se revisaron políticas, disposiciones legales y normas en las que se basan estas organizaciones para ejecutar las comprobaciones.

Las buenas prácticas de diseño centrado al usuario han ido evolucionando de manera consistente [10], y acumulan varias décadas de prácticas y perfeccionamiento. Su aplicación en el contexto cubano [11], ofrece una ventana amplia de oportunidades,

Citación: R. Cuervo, "Evaluación de la experiencia de usuario en el contexto cubano", Revista de I+D Tecnológico, vol. 15, no. 2, pp. (27-37), 2019.

Tipo de artículo: Original. Recibido: 12 de septiembre de 2018. Recibido con correcciones: 8 de mayo de 2019. Aceptado: 26 de julio de 2019.

DOI. https://doi.org/10.33412/idt.v15.2.2225

Copyright: 2019 R. Cuervo. This is an open access article under the CC BY-NC-SA 4.0 license (https://creativecommons.org/licenses/by-nc-sa/4.0/). 


\section{Experiencia de usuario}

\subsection{De la usabilidad a la experiencia de usuario}

La definición de la usabilidad ha sido sujeta a constantes reajustes. Esta se encuentra plasmada en los estándares internacionales [12]. En la ISO-9241-11 se describe en términos de efectividad, eficiencia y satisfacción en un contexto determinado.

Nuevos paradigmas de la interacción con las tecnologías continúan surgiendo y prosperando desde hace más de tres décadas, [12]. Esto demanda la revisión constante de conceptos, los cuales han expandido su alcance y ya no muestran a la usabilidad solamente como una propiedad del producto [1], también como el resultado de una interacción.

El profesor emérito de la Universidad de California Donald Norman, se especializa en la Ciencia Cognitiva. Sus aportes contribuyen al tratamiento de la definición del término experiencia de usuario [13]. Este investigador ha mencionado la relación que existe entre la usabilidad y la psicología, la cual se destaca por su importancia para el diseño, descripción o mejora sobre lo que el usuario percibe mientras interactúa con un sistema. En la ISO 9241-210:2010 [14] el concepto de la UX se define como "la percepción y respuesta resultantes del uso (anticipado o no) de un producto, sistema o producto".

La comprensión de la experiencia de usuario suele resultar difícil, debido al constante cambio de las tendencias tecnológicas, las que ejercen una gran influencia sobre las características de los productos. Por esta razón, los estudios realizados sobre el tema suelen volverse obsoletos en breves períodos de tiempo[15].

Nigel Bevan destaca la diferencia entre usabilidad y UX [16] en términos de rendimiento y placer. Cada una responde a los siguientes objetivos:

\section{Usabilidad}

- Evaluar efectividad, satisfacción y eficiencia.

- Construir una interfaz/producto fácil de utilizar.

- Mejorar el aprendizaje.

UX

- Comprender al usuario, qué hace y qué desea.

- Reconocer y sugerir respuestas relacionadas con las emociones.

\subsection{Métodos de evaluación de la UX}

Las pruebas de Usabilidad evalúan elementos como el éxito de las tareas, la satisfacción del usuario, el número de intentos o el tiempo utilizado [17]. Estas métricas deberían ser cuantificables para que puedan ser sometidas a mediciones. En el caso de la UX se toman en cuenta aspectos que requieren mayor observación [18], pero que aportan información valiosa para la toma de decisiones a través del proceso de desarrollo, los cuales permiten evitar las divagaciones basadas en instintos y suposiciones gracias al enfoque de los objetivos en metas realistas. En ambos casos resulta difícil estimar el tamaño y la magnitud del alcance de las evaluaciones.

Al inicio y durante el proceso de desarrollo de un sistema, se definen los objetivos que este debe cumplir a través de las funcionalidades. Si estas están dirigidas a aumentar la productividad, las medidas objetivas son prioritarias, pero si requieren mayor atención en la satisfacción del usuario, entonces las medidas subjetivas adquieren mayor relevancia [17].

En la ingeniería de la usabilidad se emplean cuestionarios para evaluar el nivel de satisfacción de los encuestados [18]. La recolección de estos datos constituye un aporte estratégico, antes, durante y después del lanzamiento de un producto. Contribuyen a esclarecer las metas trazadas y a encaminar el desarrollo sobre bases sólidas.

La UX suele ser interpretada como una extensión del concepto de usabilidad [12]. Aunque su concepto continúa siendo revisado, cada vez resulta más fácil encontrar definiciones que brindan mayor claridad al tema. La evolución de la ciencia y la tecnología aplicada a la interacción entre usuarios y sistemas, tiene un ritmo rápido y vertiginoso. Por esta razón, resulta necesaria una revisión constante de las definiciones de estos paradigmas.

La UX ha madurado en el transcurso de los años recientes, y ha evolucionado como un factor competitivo en el desarrollo de productos y servicios de software [5]. Sin embargo, se han encontrado dificultades en el entorno académico, debido al insuficiente enfoque sobre la realidad de las condiciones de la industria del software. Su aplicación en el marco de desarrollo industrial brinda transparencia, inspección y adaptación. Se recomienda su aplicación desde las primeras fases de desarrollo, asî como el adiestramiento al personal involucrado en la materia. De esta manera se aprovechan estilos de diseño, evaluación y control disponibles en paradigmas ágiles.

La evaluación de la UX incorpora técnicas enfocadas en factores subjetivos [19]. Este modelo vincula los 
atributos del producto a las necesidades humanas y sus valores. Su objetivo consiste en adquirir una mayor comprensión del proceso de interacción del cliente con los productos y servicios. Esto se logra permitiendo al usuario expresar sus sentimientos, impresiones y actitudes en formas fáciles e inmediatas [20].

El estudio de los métodos de evaluación de la experiencia de usuario (UXEM, del inglés user experience evaluation methods), tiene fundamentos basados en la relevancia de las necesidades psicológicas vinculadas al placer y el estímulo que perciben los usuarios durante la interacción con los productos [21]. Este acercamiento ha proliferado en investigaciones que destacan por su diversidad, lo cual complica la toma de decisiones al momento de decidir qué métodos de evaluación se ajustan mejor al producto.

Existen variantes diseñadas para mejorar esta situación. Una de ellas consiste en la identificación de un total de 89 UXEM [22] en base a los siguientes objetivos:

- Facilitar el proceso de identificación y validación de los UXEM

- Simplificar la selección del UXEM adecuado a utilizar

- Identificar las ventajas o desventajas, los UXEM se especifican para las necesidades de determinados proyectos

- Asistir a los investigadores y practicantes de la UX en la creación de nuevos UXEM que se adecúen a sus proyectos

En la región de Latinoamérica estas prácticas son tomadas en cuenta con cierta latencia [12]. Sin embargo, en el contexto brasileño estudio evaluó el estado de las prácticas de usabilidad y UX en pequeñas y medianas empresas [22]. Se demostró que muchas de estas permiten liberar versiones de prueba a sus usuarios para ser probadas, lo cual les permite anticiparse a los posibles contratiempos que pueden surgir antes del lanzamiento de versiones oficiales del producto.

Las investigaciones sobre UX se enfocan en emociones positivas como la alegría, la diversión, confianza u orgullo más que en los detalles negativos de un producto [3]. El trabajo dirigido a potenciar la aprobación del cliente, puede garantizar un lugar prevaleciente en el mercado [23]. Su importancia presiona a las compañías a integrar métodos y técnicas dirigidos a mejorar la UX.

Las compañías especializadas en la consultoría de la usabilidad han incrementado su presencia en el mundo
[24]. La Junta Internacional para la Cualificación de la Usabilidad y la Experiencia de Usuario (UXQB), es un consorcio de expertos en usabilidad y UX. Tiene como objetivo la certificación de profesionales y se ha convertido en un estándar internacional para la certificación de entidades y profesionales evaluadores de la calidad de software.

\subsection{Informatización y aseguramiento de la calidad en software en Cuba}

En Cuba se ejecutan acciones dirigidas al cumplimiento de la política integral para el perfeccionamiento de la informatización de la sociedad en cuba [7]. Esta política está apoyada sobre las bases del Plan Nacional de Desarrollo Económico y Social hasta el 2030 mencionadas en el Lineamiento 108 [25].

El Estado cubano ha declarado su interés en estos procesos, los que son prioritarios en la gestión gubernamental del país [8].

El Ministerio de Comunicaciones (MINCOM) ha sido el encargado de elaborar la política integral, como documento rector para los procesos de informatización de la sociedad de manera segura y sostenible [7]. Es aplicada dentro de un marco legal constituido por:

- 1 Decreto-Ley

- 2 Decretos

- 9 Normas complementarias

Entre las políticas específicas se encuentra "Reordenar la actividad productiva y de servicios asociada al sector", que incluye el establecimiento de un sistema de certificación para la calidad, la seguridad y la interoperabilidad [7].

El aseguramiento de la calidad de los productos y servicios tiene como objetivo el cumplimiento de estándares internacionales, debido a su importancia para el desarrollo económico del país, según el lineamiento 83 de la política económica y social [6].

En la implementación de proyectos dedicados a la proyección de estos objetivos, participan organizaciones y entidades de orígenes académicos, entidades públicas, empresariales, desarrolladores del nuevo modelo de gestión no estatal y medios de comunicación [26]. Forman parte del ecosistema de las Tecnologías de la Información y las Comunicaciones (TIC) del país y se han incluido en la creación de la Unión de Informáticos de Cuba (UIC), como organización de la sociedad civil. 
La Oficina Nacional de Normalización ha aprobado la elaboración de Normas Cubanas a través de los Comités Técnicos de Normalización. En el caso de la Norma Internacional ISO/IEC 12207, fue preparada por el Comité Técnico Conjunto ICO/IEC JTC 1, Tecnologías de la información, Subcomité SC 7, Ingeniería de software [27].

Esta norma internacional "se aplica a la adquisición de sistemas, y productos y servicios de software, así como al suministro, desarrollo, operación y mantenimiento de productos de software" [27].

La norma Cubana contiene procesos, actividades y tareas para verificar que los productos y procesos de software cumplen con sus requisitos y planes establecidos [27]. Está destinada a proveedores, desarrolladores, operadores, mantenedores, dirigentes, gestores del aseguramiento de la calidad y usuarios de productos de software.

El MINCOM emitió la Resolución 166/2017 el 21 de septiembre de 2017, que aprueba el procedimiento para la obtención del certificado que garantiza la seguridad y la calidad los sistemas contable-financieros [9].

La resolución contiene el Anexo No. 1, donde se definen los requisitos informáticos de seguridad y calidad para los sistemas contable-financieros soportados sobre las TICs.

En el apartado A se disponen diez requisitos de seguridad que mencionan el uso de trazas, mecanismos de control por roles de usuario, cifrado de la información y alertas ante intentos de violación. Resulta relevante la mención de la usabilidad en el requisito 6, donde se reconoce la importancia del diseño para la navegación intuitiva, segura y clara.

El apartado B se refiere a los requisitos informáticos de calidad y su evaluación a través de:

- Normas Cubanas vigentes sobre Ingeniería de Software y sistemas

- Requisitos de calidad y evaluación de software (SQuaRE)

- Modelos de calidad de software y sistemas

La usabilidad es mencionada como una característica de calidad a comprobar. No se definen los tipos de pruebas a realizar. Tampoco se mencionan los términos que corresponden a la UX, obviando el impacto de esta sobre la calidad y la aceptación de los productos y servicios.

\subsection{Evaluación de la UX durante la gestión de la calidad en software por entidades cubanas}

Las entidades evaluadoras y/o certificadoras de calidad en software dirigen sus servicios a desarrolladores y proveedores de productos y soluciones que forman parte de la Industria Nacional de Programas y Aplicaciones Informáticas [27]. Estas utilizan normas internacionales [27] como marco de referencia, así como disposiciones legales y regulaciones ministeriales [28] según el caso.

Para la búsqueda y análisis de los UXEMs aplicados a productos y servicios de software cubanos, se seleccionaron cuatro entidades evaluadoras tomando en cuenta los criterios:

- Objeto social.

- Experiencia en el sector.

- Fundamentos para el ejercicio de evaluación (estándares, resoluciones).

- Tipo de validación (dictamen, certificación).

\subsubsection{Centro Nacional de Calidad de software (CALISOFT)}

CALISOFT presta servicios de evaluación de productos, servicios, procesos, y el asesoramiento de especialistas, con el objetivo de promover buenas prácticas y gestionar la calidad a través de tareas de planificación, control y aseguramiento.

El centro cuenta con laboratorios destinados a la ejecución de pruebas de calidad. Algunos de estos se encuentran ubicados en la Universidad de las Ciencias Informáticas (UCI), con la que está relacionada y conectada tecnológicamente y donde también radica un departamento de calidad. Presta sus servicios a las entidades que los soliciten, a lo largo de todo el país.

Esta entidad verifica en sus evaluaciones los requisitos establecidos en el apartado 3.3.1 de la norma NC ISO/IEC 12119:2005 [28] para la instalación, presencia de funciones, corrección y consistencia. Estos requisitos de calidad están incluidos como complementarios a los sistemas informáticos y productos de software.

Para la usabilidad se basa en la norma ISO/IEC 25010:2011 [28], descritas en 6 sub-características:

- Operabilidad, define 38 requisitos para establecer los formatos y restricciones de presentación de los formularios, según el estilo y tamaño de las fuentes, los campos de entrada y los botones. Los elementos a evaluar están distribuidos según el tipo de sistema: 
- General, para todos (19 requisitos).

- Productos o sistemas para la Web (17 requisitos).

- Sistemas de gestión (1 requisito).

- Aplicaciones de escritorio (1 requisito).

- Cognoscibilidad, define 11 requisitos para referirse a cómo se busca la información en la pantalla principal, la cantidad de clics, el color de los vínculos y la velocidad del menú. Esto permite que los usuarios puedan navegar con facilidad por los contenidos del sistema. Para los productos o sistemas para la Web contiene dos requisitos.

- Reconocibilidad, contiene nueve requisitos dirigidos al del lenguaje, a través de títulos, logotipos y otros elementos que sean descriptivos y distintivos. Se recomienda el alineamiento de los contenidos para que se ajusten al perfil temático definido. Dedica cuatro indicaciones para los sistemas para la web.

- Protección ante errores de usuarios, trata el uso de las opciones por defecto en los formularios, el tratamiento de errores a través de advertencias ante eventos o acciones, y guías para la solución de problemas. La escasez de requisitos es notable, debido a que solo tiene cuatro definiciones.

- Estética de interfaz de usuario, con 18 requisitos, guarda relación con la subcaracterística de operabilidad en cuanto al uso apropiado de tipografías, la ubicación de menús, la alineación de elementos y los niveles de importancia de los contenidos. Vela por la ortografía y la composición del texto en el caso de páginas. No hace mención a la interacción del usuario con estos elementos. Divide los puntos a evaluar en:

- General, para todos (11 requisitos)

- Productos o sistemas para la Web (4 requisitos)

- Aplicaciones de escritorio (tres requisitos)

- Accesibilidad, presenta siete requisitos que definen la presentación de contenidos para personas con discapacidades físicas. En el caso de los elementos gráficos, se requiere la inclusión de un texto alternativo. Además, se exige el uso correcto del contraste entre el texto y el fondo y la identificación del cambio de idiomas para el texto. Se utilizan herramientas como Colour Contrast Analyser para determinar la legibilidad del texto y el contraste visual de los elementos (figura 1).

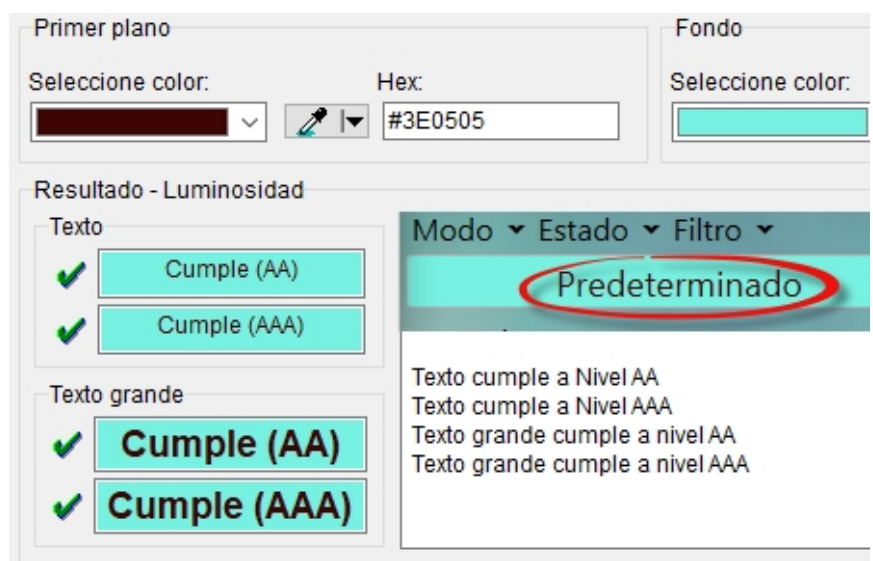

Figura 1. Análisis de contraste utilizando Colour Contrast Analyser para evaluar la usabilidad de un producto en Calisoft.

Los requisitos organizados en subcaracterísticas de usabilidad, proporcionan una guía para la evaluación de elementos de interacción entre la persona y el sistema. Se centran exclusivamente en el producto, sin incluir la participación del usuario en las evaluaciones, las cuales se realizan en entornos limitados a los especialistas. Los datos sobre la experiencia de usuario son insuficientes para evaluar los factores subjetivos que influyen en la satisfacción del cliente. No se mencionan mecanismos de selección de UXEMs que se adecúen a las características del producto, obviando la aplicación de estos.

Tabla 1. Fragmento del informe de resultado de la evaluación de calidad del producto.

\begin{tabular}{|l|l|}
\hline \multicolumn{2}{|c|}{\begin{tabular}{c}
\multicolumn{1}{|c|}{ Característica: Usabilidad } \\
No conformidades (NC)
\end{tabular}} \\
\hline NC: 29 & $\begin{array}{l}\text { No se diferencian los campos obligatorios } \\
\text { de los opcionales. Ver imágenes de la } \\
\text { 4.1.1.1.1 a la 4.1.1.1.3. Localización: } \\
\text { Gestiona clientes } \\
\text { Gestionar chofer } \\
\text { Operaciones/Recargos } \\
\text { Revisar en todo el sistema } \\
\text { Clasificación: operabilidad }\end{array}$ \\
\hline NC: 30 & $\begin{array}{l}\text { El tamaño de la caja de texto es menor que } \\
\text { la información que se recoge en ella. Ver } \\
\text { imagen 4.1 .2.1.1. Localización: } \\
\text { Facturación } \\
\text { Clasificación: operabilidad }\end{array}$ \\
\hline NC: 31 & $\begin{array}{l}\text { No se resaltan los enlaces del menú al ser } \\
\text { seleccionados. Ver imágenes 4.1.9.1.1 y } \\
\text { 4.1.9.1.2. }\end{array}$ \\
\hline
\end{tabular}




\begin{tabular}{|l|l|}
\hline & $\begin{array}{l}\text { Localización: menú principal } \\
\text { Configuración/Menú de opciones } \\
\text { Revisar en todo el sistema } \\
\text { Clasificación: operabilidad }\end{array}$ \\
\hline NC: 32 & $\begin{array}{l}\text { No se definen de manera correcta gráficos } \\
\text { y tablas utilizando sus atributos. Ver } \\
\text { imágenes 4.1.18.1.1 y 4.1.18.1.2. } \\
\text { Localización: informes } \\
\text { Estadísticas } \\
\text { Revisar en todo el sistema } \\
\text { Clasificación: operabilidad }\end{array}$ \\
\hline
\end{tabular}

\subsubsection{Empresa de aplicaciones informáticas (DESOFT)}

DESOFT ofrece servicios de desarrollo de software, distribución de productos propios y de terceros, capacitación y soporte. Tiene representaciones territoriales en todo el país.

Cada división territorial cuenta con infraestructura logística autosuficiente para la gestión comercial, desarrollo y soporte. Aunque algunos de sus productos son evaluados en CALISOFT, cuenta con departamentos de gestión de la calidad.

El Grupo de Calidad de Software de DESOFT División Habana radicado en la sede central de la empresa, es el encargado de elaborar los procedimientos para revisar las normas, y son extendidos al resto de las divisiones.

Se revisaron documentos que sirven de guía para la revisión de la usabilidad y la percepción de los clientes sobre los productos y servicios ofrecidos:

- Lista de chequeo de usabilidad.

- Anexo 3. Encuesta al cliente.

\subsubsection{Lista de chequeo de usabilidad}

Tiene como objetivo verificar el empleo de las buenas prácticas en el diseño de las interfaces gráficas. Consiste en un conjunto de preguntas agrupadas por categorías según el tipo de componente a evaluar:

- Menús: presenta seis preguntas que verifican la uniformidad de la estructura de la información, el agrupamiento de los elementos del menú y el orden lógico de estos.

- Formularios de búsqueda: consiste en 11 preguntas donde la ortografía juega un papel importante en la evaluación de la funcionalidad. Se verifica el ordenamiento de los campos de filtro y la forma en la que se muestran los resultados de la búsqueda.
- Formularios de edición: posee 28 preguntas que referencian exhaustivamente la ortografía. Se hacen distinciones en dependencia del idioma (español o inglés). También se verifican el agrupamiento de los campos, el ancho de los formularios, los iconos, el orden de los botones y la validación de los tipos de datos introducidos.

- Listas: es el último grupo de cinco preguntas dirigidas al chequeo del conjunto de datos presentados en listas. Se verifica el uso correcto de los íconos, su correspondencia con los botones y el orden de aparición de los campos que puedan ser mostrados.

Durante el análisis de las 50 preguntas contenidas en estos grupos de categorías, no se encuentran detalles que involucren al usuario. Estas están orientadas al cumplimiento estricto de parámetros normados, para la presentación de textos, opciones y el empleo de buenas prácticas en el diseño de la interfaz gráfica.

Los formularios de edición son los componentes que más análisis requieren, porque concentran la mayor cantidad de preguntas. Esto se debe al papel que juegan en el procesamiento de la información que introduce el usuario. Por esta razón, estas funcionalidades son las que producen más experiencias de interacción con el sistema. Sin embargo, no se hacen referencias al uso del color y contraste, aspectos importantes en la carga cognitiva del usuario, en un ambiente donde prevalece el uso de textos.

El área donde se aplica la lista de chequeo para la revisión de la usabilidad, ofrece oportunidades para la validación de la experiencia del usuario. Estas no son aprovechadas debido a la falta de preguntas dirigidas a obtener información subjetiva sobre la interacción del usuario con el sistema.

\subsubsection{Encuesta al cliente}

La encuesta es un documento diseñado como formulario y está dirigido a los usuarios. Contiene aspectos a valorar sobre las experiencias obtenidas con los productos y servicios que brinda la empresa. Es utilizado para medir el grado de satisfacción del cliente y está dividido en tres secciones: relacionado a los Recursos Humanos (tabla 2), al Producto (tabla 3) y al Servicio (tabla 4). Finalmente se reserva un espacio para que el encuestado pueda emitir comentarios.

Los aspectos a valorar están acompañados por una escala de puntuación. El usuario debe otorgar los puntos según su criterio sobre la calidad percibida: 2=Mal, 3=Regular, 4=Bueno, 5=Muy Bueno. En el caso del 
espacio reservado para emitir comentarios, no se incluye el sistema de puntaje. Esto difiere con lo aplicado a las secciones anteriores y no permite obtener la claridad necesaria para su procesamiento.

Tabla 2. Relacionado a los recursos humanos

\begin{tabular}{|l|l|l|l|l|}
\hline Aspectos a valorar & $\mathbf{2}$ & $\mathbf{3}$ & $\mathbf{4}$ & $\mathbf{5}$ \\
\hline $\begin{array}{l}\text { La atención de los especialistas } \\
\text { comerciales. }\end{array}$ & & & & \\
\hline $\begin{array}{l}\text { Las habilidades comunicativas de } \\
\text { nuestros consultores técnicos. }\end{array}$ & & & \\
\hline La atención de los consultores técnicos. & & & & \\
\hline $\begin{array}{l}\text { La formación y experiencia de nuestros } \\
\text { especialistas. }\end{array}$ & & & & \\
\hline
\end{tabular}

Tabla 3. Relacionado al Producto

\begin{tabular}{|l|l|l|l|l|}
\hline Aspectos a valorar & $\mathbf{2}$ & $\mathbf{3}$ & $\mathbf{4}$ & $\mathbf{5}$ \\
\hline Diseño & & & & \\
\hline Funcionalidad & & & & \\
\hline Presentación & & & & \\
\hline Calidad & & & & \\
\hline $\begin{array}{l}\text { Evolución (actualizaciones y nuevas } \\
\text { versiones) }\end{array}$ & & & & \\
\hline
\end{tabular}

Tabla 4. Relacionado al Servicio

\begin{tabular}{|l|l|l|l|l|}
\hline Aspectos a valorar & $\mathbf{2}$ & $\mathbf{3}$ & $\mathbf{4}$ & $\mathbf{5}$ \\
\hline Calidad & & & & \\
\hline Capacidad de respuesta & & & & \\
\hline Cumplimiento de los plazos previstos & & & & \\
\hline Frecuencia de visitas preventivas & & & & \\
\hline
\end{tabular}

El cuestionario ofrece una visión global de la percepción que tienen los clientes sobre los productos y servicios recibidos, pero está limitada a una escala que no brinda detalles sobre el origen de las inconformidades en los casos necesarios.

El lenguaje utilizado en el diseño de las preguntas es genérico y produce un esfuerzo cognitivo extra, lo que influye de manera negativa en la búsqueda honesta de factores subjetivos que contribuyen a la toma de acciones para mejorar la experiencia del cliente, y de esta forma su satisfacción.

El marco de aplicación de estas preguntas brinda una oportunidad valiosa de acercamiento a los usuarios. Con la selección de los UXEMs adecuados, puede ser aprovechada exitosamente.

La aplicación de esta encuesta se realiza en la culminación de las prestaciones brindadas a los clientes. Esta situación no brinda la posibilidad de comparar entre escenarios diferentes. Las etapas iniciales marcan un hito y podrían como termómetro del estado de la ejecución de los servicios.

\subsubsection{Consultoría económica de la asociación Nacional de Economistas y Contadores de Cuba (CANEC)}

CANEC es una consultoría económica líder en el mercado cubano, la cual aplica técnicas modernas de gestión a partir de la experiencia de consultores y auditores [29].

Su política de calidad está dirigida a la satisfacción de las necesidades y expectativas de sus clientes. Ofrece los servicios de asesoría, auditoría y consultoría conforme con las normas y las legislaciones aplicables.

$\mathrm{Su}$ cartera de servicios de auditorías comprende las modalidades [29]:

- Auditoría financiera con o sin dictamen.

- Auditoría financiera a sucursales extranjeras.

- Auditorías especiales:

- Asesoría de tipo contable y financiera.

- Servicios de contabilidad.

- Servicios de teneduría de libros.

- Consultoría en control interno.

- Consultoría en manuales y procedimientos.

- Consultoría en dirección y gestión empresarial.

- Consultoría en el proceso inversionista.

- Consultoría en formación de cooperativas no agropecuarias.

- Consultoría integral colaborativa.

- Servicios de asesoría y consultoría en gestión comercial y financiera, de comercio exterior y de la inversión extranjera. 
○ Entrenamientos y adiestramiento.

Para la revisión, dictamen y certificación de productos informáticos que soportan operaciones contables, se basa en la Resolución No. 340/2004 [30] del Ministerio de Finanzas y Precios, para comprobar el grado de adaptación a las Normas Cubanas de Contabilidad.

Las técnicas empleadas se aplican en etapas pactadas con los clientes. Estas consisten en:

- Revisión de documentos

- Visitas a entidades que utilizan el sistema

- Entrevistas

- Observación directa de actividades

Se analizó una muestra del Informe final del servicio de certificación de sistemas informáticos para la contabilidad, el cual sintetiza los resultados de la inspección:

- Dictamen: declara la culminación del servicio y la conformidad del producto con las normas vigentes.

- Introducción: ofrece información contractual y una breve descripción del trabajo realizado en sus etapas.

- Datos generales del sistema: caracteriza las funcionalidades evaluadas en el software.

- Conclusiones: resume la conformidad del producto mencionando las resoluciones vigentes para ello.

- Recomendaciones: opcional.

- Contenido del informe: descripción de las características evaluadas en el sistema y los fundamentos utilizados para ello.

- Dictamen e Informe gerencial: consiste en la entrega por escrito del informe y dictamen al cliente que solicitó el servicio de auditoría.

Se analizaron los procedimientos utilizados para evaluar las conformidades del sistema para ser certificado [30]. Estos se reducen a las capacidades que tiene el software de ejecutar las operaciones en correspondencia con lo estipulado en las normas y regulaciones para la contabilidad. No hacen uso de estándares ni modelos especializados en la ingeniería de software. Debido a esto la usabilidad no es un objetivo en la evaluación, y con ello la UX tampoco.

$\mathrm{Si}$ la evaluación culmina satisfactoriamente, se enuncia en el dictamen que "el producto reúne los requerimientos indispensables para su explotación con eficiencia". Este argumento resulta ambiguo e insuficiente cuando se contrastan los aspectos evaluados, con los requerimientos mínimos estipulados para certificar sistemas informáticos [9]. Tampoco garantiza el grado de satisfacción del usuario y se conforma con los requisitos mínimos asociados a la actividad contable financiera [30]. En la evidencia documental no se reflejan los resultados de las entrevistas realizadas, ni se mencionan métodos de evaluación asociados a la UX.

La actividad contable financiera ejerce gran presión sobre los profesionales del sector [31]. Esto se debe al requerimiento de altos niveles de concentración, motivación y juicio. Por esta razón, la evaluación de la UX resulta muy conveniente, para reducir la carga cognitiva producida por la complejidad de las operaciones. Situación que puede contribuir al rendimiento y consecuentemente al éxito en la utilización del software. Estos aspectos fundamentales no son objetivos de análisis en las auditorías que realiza la CANEC a productos de software.

\subsubsection{Agencia Certificadora de Protección (ACERPROT)}

ACERPROT es una agencia subordinada al Ministerio del Interior (MININT). Su objetivo consiste en la certificación y homologación de productos de seguridad con el empleo de laboratorios de referencia, y de personas vinculadas a labores de protección.

Los sistemas informáticos forman parte de los productos evaluados por la agencia. Para ello se basa en lo establecido en la Resolución 128/2011 del MINCOM [32]. Esta legislación establece, regula y controla las normas técnicas y operacionales de todas las redes informáticas y sistemas de comunicaciones en general que funcionan en el país.

La metodología de evaluación de ACERPROT contiene una exhaustiva lista de chequeos que verifica 34 requisitos y ocho subrequisitos desglosados en incisos:

- Lista de chequeo

- Contraseñas y cifrado

- Niveles de acceso

- Menús personalizables

- Perfiles, roles de usuario y grupos de restricción

- Auditoría y control de datos

- Integridad de los sistemas, ficheros y datos

- Trabajo en red y sistemas multiusuarios

- Procedimientos de salva de los ficheros de datos y el sistema

Se tomó una muestra de auditoría a un producto donde se utilizaron herramientas para la búsqueda vulnerabilidades en diferentes entornos: 
- Openvas-scanner y armitage: búsqueda de vulnerabilidades a través de los puertos usados por la aplicación.

- Edb-debugger: para intentar modificar el código del instalador y crear una instalación del sistema con una puerta trasera en Linux.

- OllyDbg: para intentar modificar el código del instalador y crear una instalación del sistema con una puerta trasera en Windows.

- SQLdict y DBPwAudit: comprobación de la calidad de las contraseñas del motor de base de datos utilizado.

La agencia juega un papel importante para el avance seguro y sostenible de la informatización del país. Su objetivo radica en la satisfacción de las directivas de seguridad según lo regulado para la seguridad informática [32], pero no se toma en cuenta el impacto de la experiencia de usuario como contribuyente en la estabilidad y fortaleza del desempeño del producto.

ACERPROT dictamina y certifica los sistemas evaluados. En este contexto se repite la situación analizada en el epígrafe anterior, respecto a la omisión de elementos regulatorios de la calidad en software y la utilización de UXEMs.

\subsection{Investigación e intención sobre la evaluación de la experiencia de usuario en el contexto cubano}

En el entorno académico cubano cuenta con espacios de intercambio entre profesionales de la ingeniería de software. Estos permiten la difusión de investigaciones que se han dirigido al aseguramiento y evaluación de la Usabilidad. Iliannis Pupo en su tesis "Procedimiento para el aseguramiento y evaluación de la usabilidad basado en patrones arquitectónicamente sensibles para los sistemas de gestión del Centro de Informatización de la Gestión de Entidades", 2012 [33], elaboró un procedimiento dividido en 3 fases vinculadas a la Ingeniería de la Usabilidad. Según el estudio, la propuesta tiene un aporte práctico importante, para la detección de problemas de usabilidad en fases tempranas del ciclo de desarrollo. En ellas se utiliza la satisfacción como un atributo elusivo y completamente dependiente de las opiniones subjetivas de los usuarios. Destaca el problema que representa la insuficiencia en la especificación de requisitos de Usabilidad, así como las limitaciones en las técnicas de la ingeniería para la captura de esta información.

Para contribuir en la solución de los problemas planteados en la investigación anterior, Yenly Pérez
Nuñez propone soluciones en su tesis "Procedimiento para evaluar la Usabilidad con la intervención del usuario", 2014 [10]. Esta investigación ofrece una guía para realizar evaluaciones de usabilidad con el usuario a un producto de software en el proceso de pruebas de aceptación. Para ello se revisaron los entornos de prueba en entidades cubanas donde se desarrolla y/o prueban softwares, en escenarios diferentes. Se extrajeron y compararon los métodos existentes, buscando la existencia de la satisfacción como uno de los objetivos. La propuesta de solución tiene lugar en los subprocesos de planificación de pruebas de aceptación y en el de ejecución de pruebas de aceptación.

El estudio sugiere que, para solucionar la necesidad de organizar las evaluaciones de usabilidad, es importante incluir la intervención de los usuarios en las pruebas.

Otro conjunto de soluciones propuestas en la tesis de Yanetsi Millet Lombida en "Procedimiento para incorporar la ingeniería de usabilidad en el proceso de desarrollo del software", 2015 [11], están encaminadas en la reducción de las No Conformidades detectadas en las evaluaciones. También coincide con la necesidad de incorporar estos procedimientos desde las primeras etapas del proceso y durante el ciclo de vida del desarrollo del software.

\section{Resultados}

La diversidad de los productos y servicios informáticos y su acelerado desarrollo, han acentuado la necesidad de la gestión de su calidad y a la vez de profundizar en los métodos utilizados para ello.

En esta investigación se revisaron 17 artículos relacionados con la UX, así como tres tesis de procedimientos (figura 2). Sus textos demuestran que la existencia de los UXEM ha dejado de ser una realidad peculiar y experimental, para insertarse seriamente en las prácticas de las metodologías de desarrollo. Su impacto es reconocido por organizaciones y corporaciones que se encuentran en la búsqueda constante de la adecuación de estos paradigmas a la realidad del estado que plantean las ciencias informáticas.

La ergonomía y el tiempo de aprendizaje influyen en la percepción del cliente hacia los productos y servicios. Las aplicaciones de los UXEM contribuyen a perfilar el tratamiento de estos aspectos. Su desarrollo tiene más de tres décadas de evolución, aunque se limita a regiones de 
marcada diferencia en aspectos de desarrollo económico, tecnológico y social.

En el caso de Cuba, los procesos encaminados a la informatización requieren la actualización y adaptación de legislaciones y normas, para la inclusión de los UXEM en el contexto nacional.

Se estudiaron los procedimientos de comprobación de la calidad en cuatro entidades evaluadoras, de las cuales 2 certifican los resultados de las evaluaciones (tabla 5). CALISOFT se destaca por ofrecer la mayor cantidad de aspectos y herramientas utilizados en el análisis de los productos.

Tabla 5. Entidades evaluadoras analizadas

\begin{tabular}{|l|c|l|l|c|}
\hline Entidad & Año & \multicolumn{1}{|c|}{ Objetivos } & \multicolumn{1}{c|}{ Bases } & Certifica \\
\hline Canec & 1979 & $\begin{array}{l}\text { Aplicación de } \\
\text { técnicas } \\
\text { modernas de } \\
\text { gestión } \\
\text { económica a } \\
\text { partir de la } \\
\text { experiencia de } \\
\text { consultores y } \\
\text { auditores. }\end{array}$ & $\begin{array}{l}\text { Resoluci } \\
\text { ono/2009 }\end{array}$ & Sí \\
\hline Acerprot & 1997 & $\begin{array}{l}\text { Certificación y } \\
\text { homologación } \\
\text { de productos } \\
\text { de seguridad. }\end{array}$ & $\begin{array}{l}\text { Decreto } 168 \\
\text { de 1998 }\end{array}$ & Sí \\
\hline Desoft & 2004 & $\begin{array}{l}\text { Servicios de } \\
\text { desarrollo, } \\
\text { distribución y } \\
\text { soporte de } \\
\text { software. }\end{array}$ & $\begin{array}{l}\text { Grupo } \\
\text { de } \\
\text { calidad } \\
\text { de } \\
\text { software } \\
\text { de } \\
\text { Desoft }\end{array}$ & No \\
\hline Calisoft & 2012 & $\begin{array}{l}\text { NC } \\
\text { Promover } \\
\text { buenas } \\
\text { prácticas y } \\
\text { gestionar la } \\
\text { calidad de } \\
\text { software. }\end{array}$ & $\begin{array}{l}\text { ISO/IEC } \\
12119: 2 \\
005\end{array}$ & No \\
\hline
\end{tabular}

Aunque existen mecanismos para la regulación, planificación, control y mantenimiento de la calidad, continúan siendo insuficientes en cuestiones de alcance. Las organizaciones destinadas a la evaluación de estos aspectos carecen en sus fundamentos, de la actualización en la práctica de sus procedimientos.

En el caso de la industria, los productos que se comercializan son analizados tomando en cuenta el cumplimiento de las tareas para las cuales fueron diseñados. Para ello no se toman en cuenta los beneficios que ofrece la participación de los usuarios, cuya actuación resulta clave en el éxito de los sistemas.

\section{Conclusiones}

La adopción de los procedimientos propuestos por los UXEMs, contribuye al acercamiento del desarrollador y evaluador a los intereses del cliente, y de esta forma, a elevar el nivel de aceptación de los usuarios. Estos métodos proporcionan herramientas que facilitan el establecimiento de metas según condiciones más realistas, y contribuyen a la reducción del tiempo y los costos durante el proceso.

El éxito de los productos y servicios tiene relación directa con la evaluación de la satisfacción del usuario. Las expectativas e impresiones de los clientes son importantes y deben ser tomadas en cuenta, incluso antes de iniciar el desarrollo o la implementación de las soluciones informáticas.

En Cuba se toman medidas para potenciar la calidad en la industria, y la compatibilidad con los estándares internacionales para impulsar el mercado nacional y la exportación. Estas políticas se han propuesto y evaluado en congresos, legislaciones y normas. Sin embargo, las inflexibilidades de las prácticas utilizadas persisten y afectan la aplicación de técnicas de medición efectivas para distintos escenarios y tipos de productos. Las entidades desarrolladoras, evaluadoras y certificadoras se hacen eco de estas disposiciones, aplicando de forma fragmentada las indicaciones.

Investigaciones como la presente, están dirigidas al contexto cubano y proponen la inclusión de buenas prácticas y la apertura al convulso pero evolutivo ritmo de la ingeniería de la usabilidad y la UX.

\section{Agradecimiento}

La inspiración y desarrollo de este trabajo se debe a la colaboración de colegas y amigos a quienes agradezco: Maité Basulto Socorro, Axel Castro Campos, Yaimí Trujillo Casañola y Alionuska Velázquez Cintra. 


\section{Referencias}

[1] N. Bevan, J. Carter, and S. Harker, "ISO 9241-11 revised: What have we learnt about usability since 1998?," Human Computer Interaction, p. 11, 2015.

[2] N. Bevan, "The evaluation of accessibility, usability and user experience," The Universal Access Handbook, C Stepanidis (ed), CRC Press, 2009.

[3] P. G. Zimmermann, "Beyond Usability - Measuring Aspects of User Experience," SWISS FEDERAL INSTITUTE OF TECHNOLOGY ZURICH, p. 113, 2008.

[4] C. Lallemand, G. Gronier, and V. Koenig, "User experience: A concept without consensus? Exploring practitioners' perspectives through an international survey," Computers in Human Behavior, vol. 43, pp. 35-48, 2015.

[5] T. Øvad and L. B. Larsen, "How to reduce the UX bottleneck train your software developers," Behaviour \& Information Technology, vol. 35, no. 12, pp. 1080-1090, 2016.

[6] P. C. d. Cuba, "Lineamientos de la Política Económica y Social del Partido y la Revolución," VI Congreso del Partido Comunista de Cuba, p. 41, 2011.

[7] M. d. C. d. Cuba, "Politica Integral para el perfeccionamiento de la Informatizacion de la sociedad en Cuba," 2017.

[8] Granma, "Cuba sin pausas en el propósito de la informatización," Órgano Oficial del Partido Comunista de Cuba, vol. 237, 2018.

[9] M. d. Comunicaciones, "Resolución No. 166/2017," Gaceta Oficial, vol. 2017, no. 40, p. 9, 2017.

[10] Y. P. Nuñez, "Procedimiento para evaluar la usabilidad con la intervención del usuario," UNIVERSIDAD DE LAS CIENCIAS INFORMÁTICAS, p. 99, 2014.

[11] Y. M. Lombida, "Procedimiento para incorporar la Ingeniería de Usabilidad en el proceso de desarrollo del software," Universidad de las Ciencias Informáticas, p. 103, 2015.

[12] C. Rusu, "Usability versus User Experience: Theory and Practice," Pontificia Universidad Católica de Valparaíso (Chile), p. 12, 2014.

[13] N. N. Group (2018, Feb), "Donald Norman.". [Online]. Available: https://www.nngroup.com/people/don-norman/ [Feb. 11, 2018]

[14] ISO, "INTERNATIONAL STANDARD ISO 9241-210, Ergonomics of human-system interaction. Part 210: Humancentred design for interactive systems," ISO 2010, 2010.

[15] Z. Hasan and R. C. Gope, "Dynamics of User Experience (UX)," International Journal of Computer Applications, vol. 81, no. 16, 2013.

[16] N. Bevan, "What is the difference between the purpose of usability and user experience evaluation methods," UXEM'09 Workshop, INTERACT 2009, Uppsala, Sweden, p. 4, 2009.

[17] J. R. Lewis, "IBM computer usability satisfaction questionnaires Psychometric evaluation and instructions for use," International Journal of Human-Computer Interaction, vol. 7, no. 1, p. 23, 1995.

[18] G. Bilen, "Experience Design and Measuring User Experiences for Single Page Application," Helsinki Metropolia University of Applied Sciences, p. 52, 2016.
[19] C. Rico-Olarte, D. M. López, and S. Kepplinger, "Towards a Conceptual Framework for the Objective Evaluation of User Experience," vol. 10918, pp. 546-559, 2018.

[20] M. Schrepp, A. Hinderks, and J. r. Thomaschewski, "Design and Evaluation of a Short Version of the User Experience Questionnaire (UEQ-S)," International Journal of Interactive Multimedia and Artificial Intelligence, vol. 4, no. 6, p. 103, 2017.

[21] C. Lallemand, V. Koenig, and G. Gronier, "How relevant is an expert evaluation of user experience based on a psychological needs-driven approach?," pp. 11-20, 2014.

[22] S. Rajeshkumar, R. Omar, and M. Mahmud, "Taxonomies of User Experience (UX) Evaluation Methods," 3rd International Conference on Research and Innovation in Information Systems 2013 (ICRIIS'13), p. 6, 2013.

[23] R. Alves, P. Valente, and N. J. Nunes, "The state of user experience evaluation practice," NordiCHI '14, pp. 93-102, 2014.

[24] UXQB (2018, Feb.). "Certified Professional for Usability and User Experience-Foundation Level." [Online]. Available: https://uxqb.org/en/about-uxqb/ [Feb. 11, 2018].

[25] A. N. d. P. Popular, "Documentos del 7mo. Congreso del Partido aprobados por el III Pleno del Comité Central del PCC el 18 de mayo de 2017 y respaldados por la Asamblea Nacional del Poder Popular el 1 de junio de 2017," Pleno del Comité Central del PCC, vol. 7, 2017.

[26] T. D. Fernández, "Cibersociedad: un concepto emergente desde la Unión de Informáticos de Cuba," Unión de Informáticos de Cuba, 2018.

[27] ISO/IEC, "Norma Cubana 12207_2007," ISO, 2006.

[28] Calisoft, "REQUISITOS DE LA CALIDAD PARA SISTEMAS INFORMÁTICOS Y PRODUCTOS DE SOFTWARE," no. 01:03/2017, 2017.

[29] CANEC (2018, Feb.), "CANEC." [Online]. Available: www.canec.cu [Feb 11, 2018].

[30] M. d. F. y. Precios and MFP, "Resolución No. 340/2004, Procedimiento para dictaminar sobre el grado de adaptación a las Normas Contables Cubanas de los Sistemas Contables Financieros soportados sobre las tecnologías de la información," Gaceta Oficial, 2004.

[31] A. Ozkana, M. Ozdevecioglub, Y. Kayac, and F. Ö. Koc, "Effects of mental workloads on depression-anger symptoms and interpersonal sensitivities of accounting professionals," Elsevier España, 2014.

[32] M. d. 1. C. d. Cuba, "RESOLUCIÓN No. 128/2011, Reglamento redes privadas de datos," Gaceta Oficial, 2011.

[33] I. P. Leyva, "Procedimiento para el aseguramiento y evaluación de la usabilidad basado en patrones arquitectónicamente sensibles para los sistemas de gestión del Centro de Informatización de la Gestión de Entidades," Universidad de las Ciencias Informáticas, 2012. 